\title{
Matter-wave localization in a random potential
}

\author{
Yongshan Cheng ${ }^{1,2, *}$ and S. K. Adhikari ${ }^{1, \dagger}$ \\ ${ }^{1}$ Instituto de Física Teórica, UNESP - Universidade Estadual Paulista, 01.140-070 São Paulo, São Paulo, Brazil \\ ${ }^{2}$ Department of Physics, Hubei Normal University, Huangshi 435002, People's Republic of China
}

(Received 2 June 2010; published 26 July 2010)

\begin{abstract}
By numerical and variational solutions of the Gross-Pitaevskii equation, we studied the localization of a noninteracting and weakly interacting Bose-Einstein condensate (BEC) in a disordered cold atom lattice and a speckle potential. In the case of a single BEC fragment, the variational analysis produced good results. For a weakly disordered potential, the localized BEC's are found to have an exponential tail as in the weak Anderson localization. We also investigated the expansion of a noninteracting BEC in these potentials. We find that the BEC will be locked in an appropriate localized state after an initial expansion and will execute breathing oscillation around a mean shape when a BEC at equilibrium in a harmonic trap is suddenly released into a disorder potential.
\end{abstract}

DOI: 10.1103/PhysRevA.82.013631

PACS number(s): 03.75.Nt, 64.60.Cn

\section{INTRODUCTION}

Since the experimental realization of the Anderson localization of a Bose-Einstein condensate (BEC) in a disorder potential [1,2], this topic has been the subject of intense theoretical and experimental activities. Billy et al. [1] observed the exponential tail of the spatial density distribution when a ${ }^{87} \mathrm{Rb}$ $\mathrm{BEC}$ was released into a one-dimensional (1D) waveguide in the presence of a controlled disorder created by a laser speckle. Roati et al. [2] employed a 1D quasiperiodic bichromatic optical lattice to observe the Anderson localization of a noninteracting ${ }^{39} \mathrm{~K}$ BEC. Experimental studies employed the bichromatic optical lattice [2,3], shaken optical lattice [4], cold atom lattice [5], and speckle potentials [1]. (The effect of a repulsive atomic interaction on localization has also been studied [6].) Theoretical studies employed the bichromatic optical lattice [7-9], cold atom lattice [10,11], and random [12-14] potentials among others.

One can produce quasiperiodic [15] or random [16-18] potentials for the Anderson localization by optical means. A bichromatic optical lattice is realized by a primary lattice perturbed by a weak secondary lattice with incommensurate wavelength [2]. Random speckle potentials are produced when light is reflected by a rough surface or transmitted by a diffusive medium [19]. In addition to optical means, disordered or quasidisordered potentials could also be created by using atomic mixtures [10] or inhomogeneous magnetic fields [20,21]. Gavis and Castin [10] proposed a controlled way of producing a disordered potential by using a mixture of two different atomic species. In this approach, known as the cold atom lattice, the atoms of one of the two species, named "scatterers," are randomly trapped in the sites of an optical lattice. If the filling factor is much less than unity, only some of the sites will be occupied by one atom and the others will be empty. The atoms of the second species that could be weakly affected by the presence of the optical lattice and denoted as "test particles," feel the collisional interaction with the randomly distributed scatterers. The collisional interaction will act as a random potential for the test particles.

\footnotetext{
*yong_shan@163.com

†adhikari@ift.unesp.br,www.ift.unesp.br/users/adhikari
}

Here, with the variational and numerical solutions of the Gross-Pitaevskii (GP) equation, we investigate the localization of cigar-shaped BEC's in a 1D random speckle potential and in a disordered cold atom lattice. The random speckle potential is modeled by taking identical Gaussian spikes distributed randomly [12]. In this case the spikes may overlap. The cold atom lattice is modeled by taking a periodic distribution of nonoverlapping spikes from which a small number of spikes are taken out randomly [10]. We examine separately two regions of the localized BEC's. The first region corresponds to the center, where the density profile of the localized BEC is quite similar to a Gaussian shape. In this region, we also use the variational approximation for some analytical understanding of the localized state. The second region corresponds to the tails of the BEC, where we focus on spatially extended wave functions with exponential decay corresponding to a weak Anderson localization [12,19]. The stability of the localized state is also investigated in both cases. To the best of our knowledge this is the first detailed study of Anderson localization in the presence of only a disorder speckle or cold atom lattice potential. Most of the previous studies [12,22] employed such a disorder potential superposed on a harmonic potential.

In Sec. II we present a brief account of the 1D GP equation, the disordered potentials, and a variational analysis of the GP equation. In Sec. III we present numerical results using the split-step Fourier spectral method and compare these with a variational analysis. In Sec. IV we present a brief summary.

\section{ANALYTICAL CONSIDERATION}

We assume that the trapping potential $V(x)$ is disordered along the longitudinal $x$ direction with a strong harmonic trap in transverse directions. Then it is appropriate to consider a 1D reduction of the three-dimensional GP equation by freezing the transverse dynamics of the BEC to the respective ground states and integrating over the transverse variables [23]. The BEC dynamics of $N$ atoms can then be described by the following 1D equation for wave function $u \equiv u(x, t)[23,24]$

$$
i \frac{\partial u}{\partial t}=-\frac{1}{2} \frac{\partial^{2} u}{\partial x^{2}}+g|u|^{2} u+V(x) u,
$$


with nonlinearity $g=2 a N / a_{\perp}^{2}$, normalization $\int_{-\infty}^{\infty}|u|^{2} d x=$ 1 , and $a$ the atomic scattering length. The spatial variable $x$, time $t$, and energy are expressed in transverse harmonic oscillator units $a_{\perp}=\sqrt{\hbar /(m \omega)}, \omega^{-1}$, and $\hbar \omega$, where $m$ is the mass of an atom and $\omega$ is the angular frequency of the transverse trap.

The trap $V(x)$ can be modeled by a set of $S$ identical spikes randomly distributed along the $x$ axis [12]

$$
V(x)=V_{0} \sum_{j=1}^{S} v\left(x-x_{j}\right),
$$

where $V_{0}$ is the strength of the spike and $v\left(x-x_{j}\right)$ represents the potential of a single spike at position $x_{j}$. We consider Gaussian spikes [12]

$$
v(x)=(\sigma \sqrt{\pi})^{-1} \exp \left(-\frac{x^{2}}{\sigma^{2}}\right),
$$

with normalization $\int v(x) d x=1$ and width $\sigma$. The statistical average of the disordered potential (2) is [12]

$$
\langle V(x)\rangle \equiv \frac{1}{2 L} \int_{-L}^{L} V(x) d x=\frac{V_{0}}{D},
$$

with $D$ the average spacing between spikes and $2 L$ the spatial extension of $V(x)$. The autocorrelation function for potential $V(x)$ is defined as [12]

$$
C(d)=\langle V(x) V(x+d)\rangle-\langle V(x)\rangle^{2},
$$

where $\langle\cdot\rangle$ represents averaging as in Eq. (4). An average spike height $V_{S}$ is defined by [12]

$$
V_{S}=\left\{\frac{1}{2 L} \int_{-L}^{L} d x[V(x)-\langle V(x)\rangle]^{2}\right\}^{1 / 2} .
$$

In the presence of strong disorder a multifragmented BEC state is expected [13]. Under appropriate conditions, however, the shape of localized states may be a single fragment, so the variational treatment will be useful [25]. The variational approach allows us to obtain useful relations among the localized state parameters. We consider the stationary form $\phi(x)$ of the localized state given by $u(x, t)=\exp (-i \mu t) \phi(x)$, with $\mu$ the chemical potential. The real wave function $\phi(x)$ obeys

$$
\mu \phi(x)+\phi^{\prime \prime}(x) / 2-g \phi^{3}(x)-V(x) \phi(x)=0,
$$

where the prime denotes space derivative. We use the off-center variational Gaussian ansatz

$$
\phi(x)=\frac{1}{\pi^{1 / 4}} \sqrt{\frac{\mathcal{N}}{w}} \exp \left[-\frac{\left(x-x_{0}\right)^{2}}{2 w^{2}}\right],
$$

with $w$ the width, $x_{0}$ the center, and $\mathcal{N}$ the normalization of the localized BEC. The Lagrangian of the system is

$$
\begin{gathered}
\mathcal{L}=\int_{-\infty}^{\infty}\left[\mu \phi^{2}-\left(\phi^{\prime}\right)^{2} / 2-g \phi^{4} / 2-V(x) \phi^{2}\right] d x-\mu \\
=\mu(\mathcal{N}-1)-\frac{\mathcal{N}}{4 w^{2}}-\frac{g \mathcal{N}^{2}}{2 \sqrt{2 \pi} w}-\sum_{j=1}^{S} \mathcal{N} \mathcal{L}_{j}, \\
\mathcal{L}_{j} \equiv \frac{V_{0}}{\sqrt{\pi\left(\sigma^{2}+w^{2}\right)}} \exp \left[-\frac{\rho_{j}^{2}}{\sigma^{2}+w^{2}}\right],
\end{gathered}
$$

where $\rho_{j}=x_{0}-x_{j}$. The Euler-Lagrange equation $\partial \mathcal{L} / \partial \mu=$ 0 yields the normalization $\mathcal{N}=1$. We use it in the following equations. The remaining equations $\partial \mathcal{L} / \partial w=\partial \mathcal{L} / \partial \mathcal{N}=0$ yield, respectively,

$$
\begin{gathered}
1=-\frac{g w}{\sqrt{2 \pi}}+\frac{2 w^{4}}{\left(\sigma^{2}+w^{2}\right)} \sum_{j=1}^{S}\left(\frac{2 \rho_{j}^{2}}{\sigma^{2}+w^{2}}-1\right) \mathcal{L}_{j}, \\
\mu=\frac{1}{4 w^{2}}+\frac{g}{w \sqrt{2 \pi}}+\sum_{j=1}^{S} \mathcal{L}_{j},
\end{gathered}
$$

and determine the width $w$ and the chemical potential $\mu$. The corresponding energy $E=\int_{-\infty}^{\infty}\left[\left(\phi^{\prime}\right)^{2} / 2+g \phi^{4} / 2+\right.$ $\left.V(x) \phi^{2}\right] d x$ is given by

$$
E=\frac{1}{4 w^{2}}+\frac{g}{2 w \sqrt{2 \pi}}+\sum_{j=1}^{S} \mathcal{L}_{j}
$$

\section{NUMERICAL RESULTS}

We perform the numerical integration of GP equation (1) employing the imaginary-time or real-time split-step Fourier spectral method with space step 0.04, time step 0.001, and an initial input Gaussian pulse. The time evolution is continued till convergence. We checked the accuracy of the results by varying the space and time steps and the total number of space and time steps. Although we use a time-dependent approach, the localized states are stationary.

\section{A. A speckle potential}

To generate the speckle potential we use a set of random numbers with the standard MATLAB function RAND [12]. These numbers are then mapped into the interval $[-L, L]$ by a linear transformation and denote the position $\left(x_{j}\right)$ of the spikes. We select $S=300, L=30$, and a small width $\sigma=0.1$. The spikes are described by Eq. (3) and the speckle potential by Eq. (2). A typical speckle potential $V(x)$ for $V_{0}=1$ is plotted in Fig. 1(a). The statistical average of Eq. (4) is $\langle V\rangle=5$. Using Eq. (6) the average speckle height is $V_{S}=4.3519$, and the spatial autocorrelation function (5) is shown in Fig. 1(b) where we also show the numerical fit [12]

$$
C(d) \approx V_{R}^{2} \exp \left(-d^{2} / \sigma_{R}^{2}\right)
$$

with amplitude $V_{R}=4.2872$ and correlation length $\sigma_{R}=$ 0.128 . We study localization with potential $V(x)$ of Fig. 1(a) for different $V_{0}$. This potential can produce a stationary BEC near $x=0$. We also repeated our studies with different speckle potentials generated with the same method and parameters as $V(x)$ but by different random processes. Similar conclusions are obtained in all cases except that the center of the localized states can be different.

We first investigate the central region of the localized BEC, and the numerical and variational results are exhibited in Figs. 2(a) and 2(b) where the BEC center $x_{0}$ is obtained by a Gaussian fitting of the numerical density profile. In Fig. 2(a) we present the density profiles $|u|^{2}$ of the localized BEC for different $V_{0}$. Good agreement between the variational and numerical densities is obtained. To understand the effect 

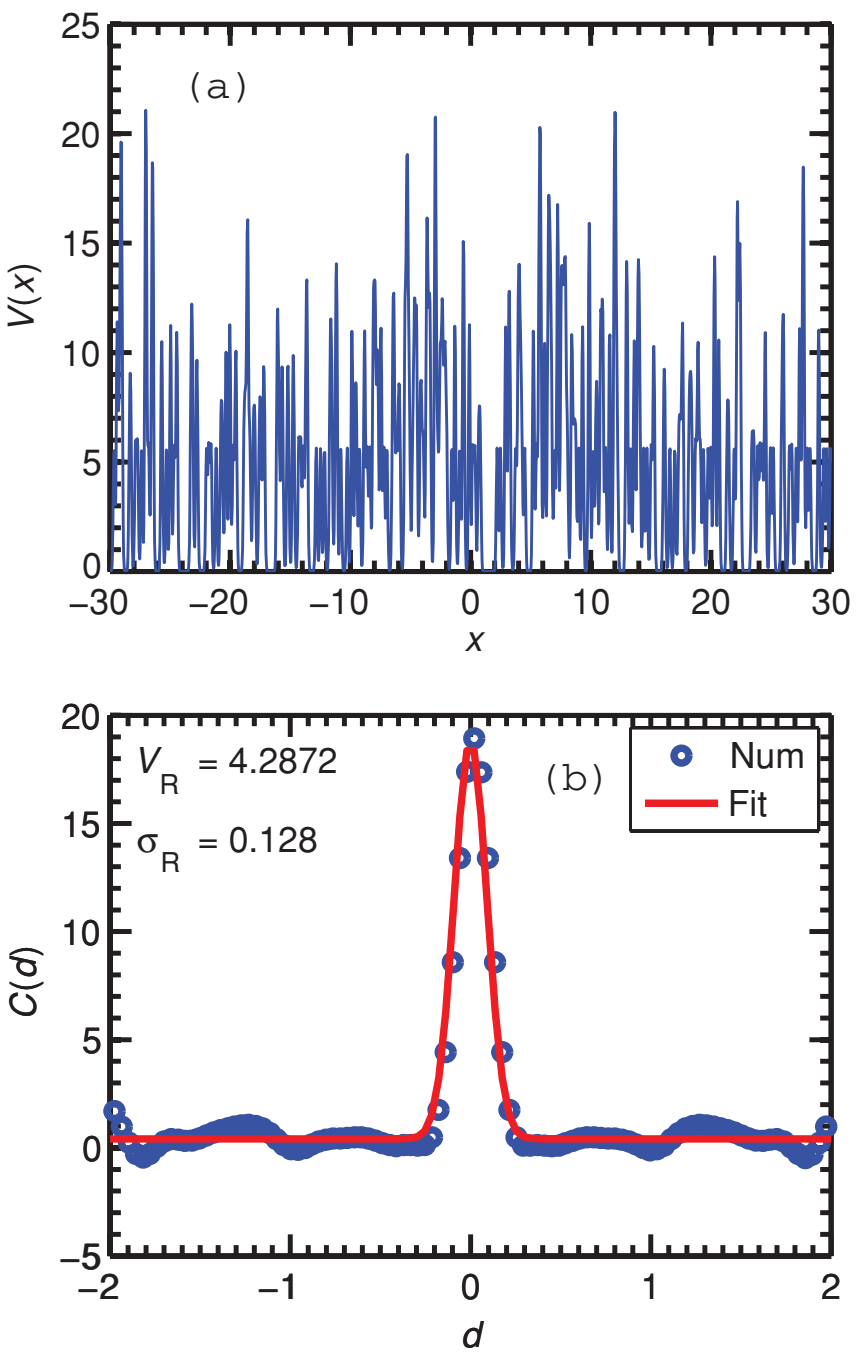

FIG. 1. (Color online) (a) Typical disordered speckle potential from Eqs. (2) and (3) with $S=300, L=30, V_{0}=1$, and $\sigma=0.1$. (b) The autocorrelation function (5) of the potential (a). The circles (Num) denote numerical results from Eq. (5) and the solid line (Fit) is the Gaussian fit (14) with $V_{R}=4.2872$ and $\sigma_{R}=0.128$.

of $V_{0}$, the numerical and variational widths (upper panel) and the chemical potential $\mu$ and $V_{S} / \mu$ (lower panel) of the localized BEC versus $V_{0}$ are plotted in Fig. 2(b) for $g=0$. (The numerical width is the root mean square size of the BEC.) Figure 2(b) shows that the width decreases and the chemical potential increases with the increase of $V_{0}$. However, the rate of change is smaller when $V_{0}$ is large enough (for example, $\left.V_{0}>0.4\right)$. It means that the kinetic energy dominates in the regime. The quantity $V_{S} / \mu$ gives a measure of disorder. However, the values of $V_{S} / \mu$ cannot be directly compared with those in Ref. [12] where a harmonic potential is used together with the disorder potential, so that a smaller disorder may lead to localization because of the additional harmonic potential.

Next we consider the effects of a small nonlinearity $g$ on the localized BEC in the case of a stronger disorder (viz. $\left.V_{0}=1\right)$. The width, energy, and chemical potential of the localized BEC versus $g$ are plotted in Figs. 3(a) and 3(b). The width, energy, and chemical potential increase with increasing nonlinearity. Increasing nonlinearity means increasing repulsion and hence
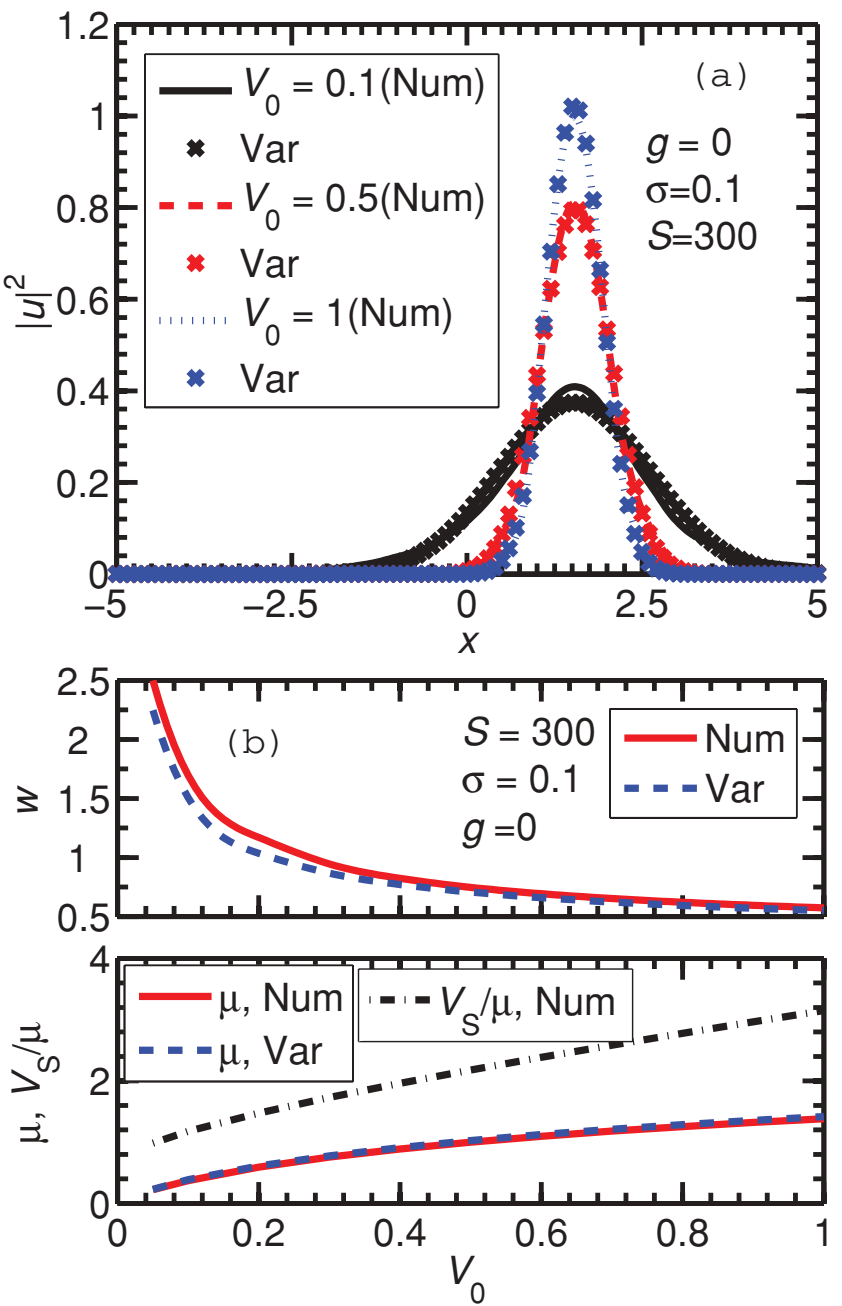

FIG. 2. (Color online) (a) The numerical (Num) and variational (Var) densities $|u|^{2}$ of the localized BEC versus $x$ for different $V_{0}$. (b) The numerical and variational widths $w$, chemical potential $\mu$ and $V_{S} / \mu$ of the BEC versus $V_{0}$.

increased width. Increasing nonlinearity also means a larger positive contribution to the Hamiltonian and hence a larger energy and chemical potential.

So far we investigated the central region of the localized BEC. In the presence of strong disorder, we also studied the tail region of the BEC but did not find an exponential decay. However, one of the more interesting issues of localization is in the presence of a weak disorder when the system is localized due to the disordered nature of the potential but not due to the strength of the potential. In the presence of a weak disorder, Anderson-like localization is expected in the tail region of the BEC. The weak disorder of a speckle potential can be acquired in two ways. One way is to reduce the strength $V_{0}$ in the potential $V(x)$. Another way is to reduce the disorder of the spikes' position. (This second case will be discussed in the following section). First, we study numerically the tail region of the BEC in potential $V(x)$ with smaller $V_{0}$. Figure 4(a) shows the effects of $V_{0}$ on the tail region of the BEC for $g=0$. The hatched lines are the exponential fitting to the tails by $\exp \left(-\left|x-x^{\prime}\right| / l\right)$. Where $l$ is the localization length $[1,12]$ and $x^{\prime}$ is the center 

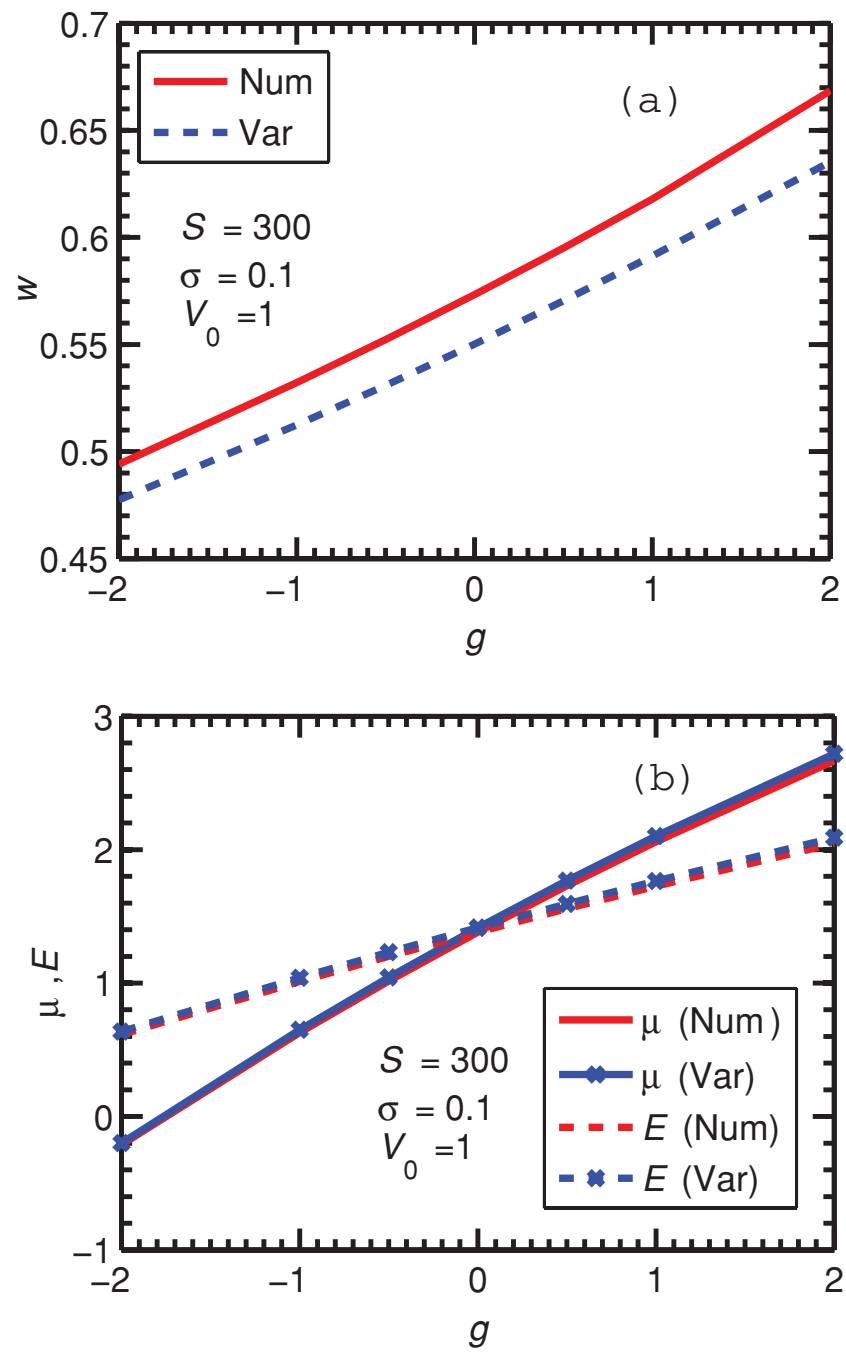

FIG. 3. (Color online) The effects of $g$ on the center region of the BEC. (a) The width and (b) the energy $E$ and chemical potential $\mu$ versus $g$.

of the exponential function. The density profile obtained numerically is found to have a clean exponential tail for smaller $V_{0}$. When $V_{0}$ is large enough, the exponential tail fades away and the tail region approximates the central Gaussian distribution. We also find that the tail is modulated by the speckle potential if $V_{0}$ is small enough. In general, the center of the exponential function is different from the center of the localized state. Also, the density profile of the tail is asymmetric around the central position because of the asymmetry of the disordered potential. Thus, the localization length for the left tail $\left(l_{L}\right)$ is different from that for right tail $\left(l_{R}\right)$. The stronger the disorder is, the smaller the localization length is. For $V_{0}<0.2$ or for $V_{S}<0.86$ corresponding to $V_{S} / \mu<1.5$ [viz. Fig. 2(b)], we have weak Anderson localization for $g=0$. These values of $V_{S} / \mu$ are larger than the $V_{S} / \mu<0.3$ considered in Ref. [12]. This is reasonable, as in Ref. [12] the authors consider a speckle potential superposed on a harmonic potential.

We study numerically the effect of the small nonlinearity $g$ on the localization of BEC as well. The results are presented in Fig. 4(b), where the numerical width $w$ (corresponding to the
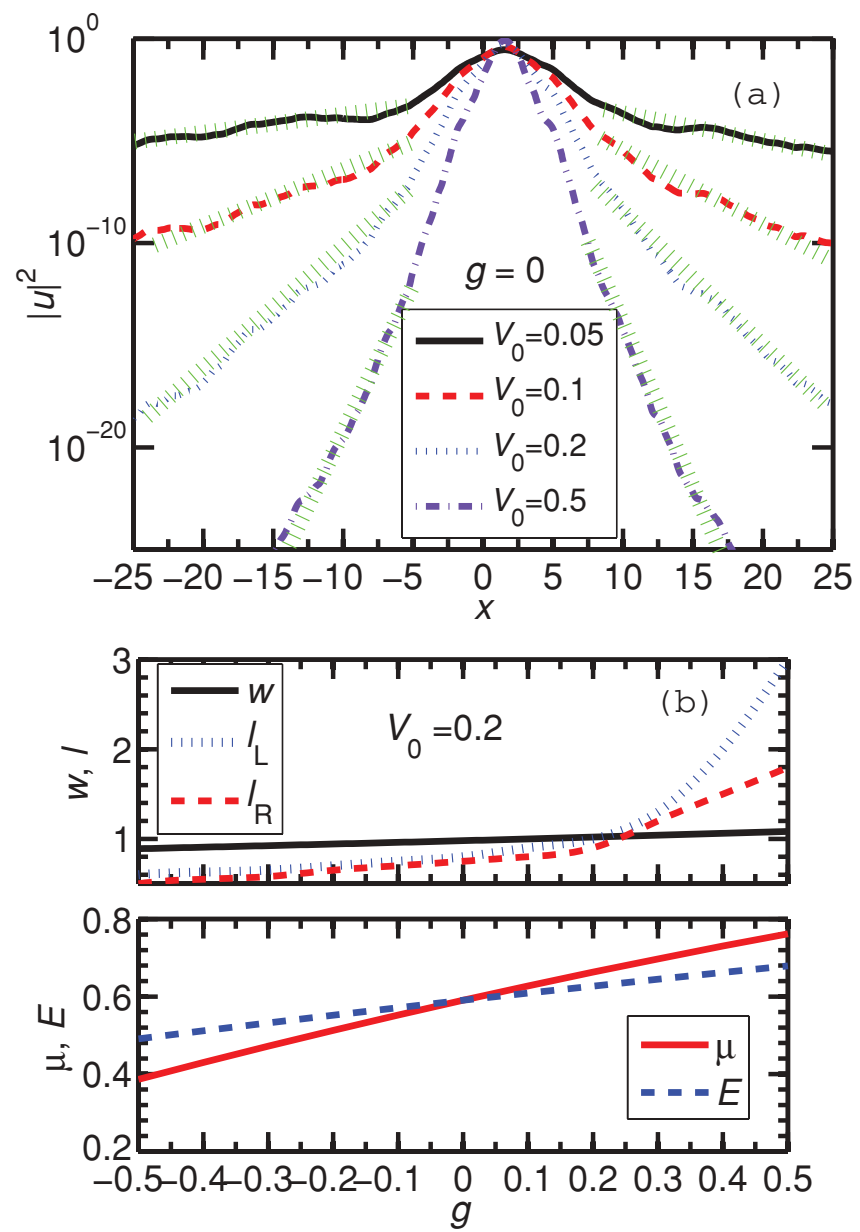

FIG. 4. (Color online) (a) The numerical density $|u|^{2}$ and its exponential fit (hatched line) versus $x$ for different $V_{0}$ and $g=0$. (b) (upper panel) The numerical width $w$ and the localiztion lengths $l_{L}$ (left tail) and $l_{R}$ (right tail) versus the nonlinearity $g$ for $V_{0}=0.2$. (lower panel) The numerical chemical potential $\mu$ and energy $E$ versus $g$ for $V_{0}=0.2$.

center region) and the localiztion lengths $l_{L}$ (left tail) and $l_{R}$ (right tail) versus nonlinearity $g$ are shown in the upper panel. When $g$ is smaller, the two localization lengths are similar as shown in the upper panel in Fig. 4(b). If $g$ is larger, the asymmetry between the two tails becomes larger. The localization lengths increase sharply with $g$ for $g>0.3$. The localization is destroyed and the BEC becomes a multifragmented state if $g>0.5$ and $V_{0}=0.2$. Compared with Fig. 3(b), the lower panel in Fig. 4(b) shows that the energy is smaller when $V_{0}$ is smaller. The result is in accordance with Fig. 2(b).

We also studied the expansion of a noninteracting BEC in the speckle potential. We start from a BEC at equilibrium in the harmonic trap. At time $t=0$, the harmonic trap is suddenly changed into a weak speckle potential as in the experiment of Billy et al. [1]. The evolution for the density profile of the BEC is shown in Fig. 5(a) where the initial expansion and the consequent breathing oscillation of the BEC are illustrated. During the evolution, the BEC remains localized and oscillates around an equilibrium point. To understand this behavior, the time evolution of the width of the BEC is plotted in Fig. 5(b) for several $V_{0}$ corresponding to a weak disorder. During the 

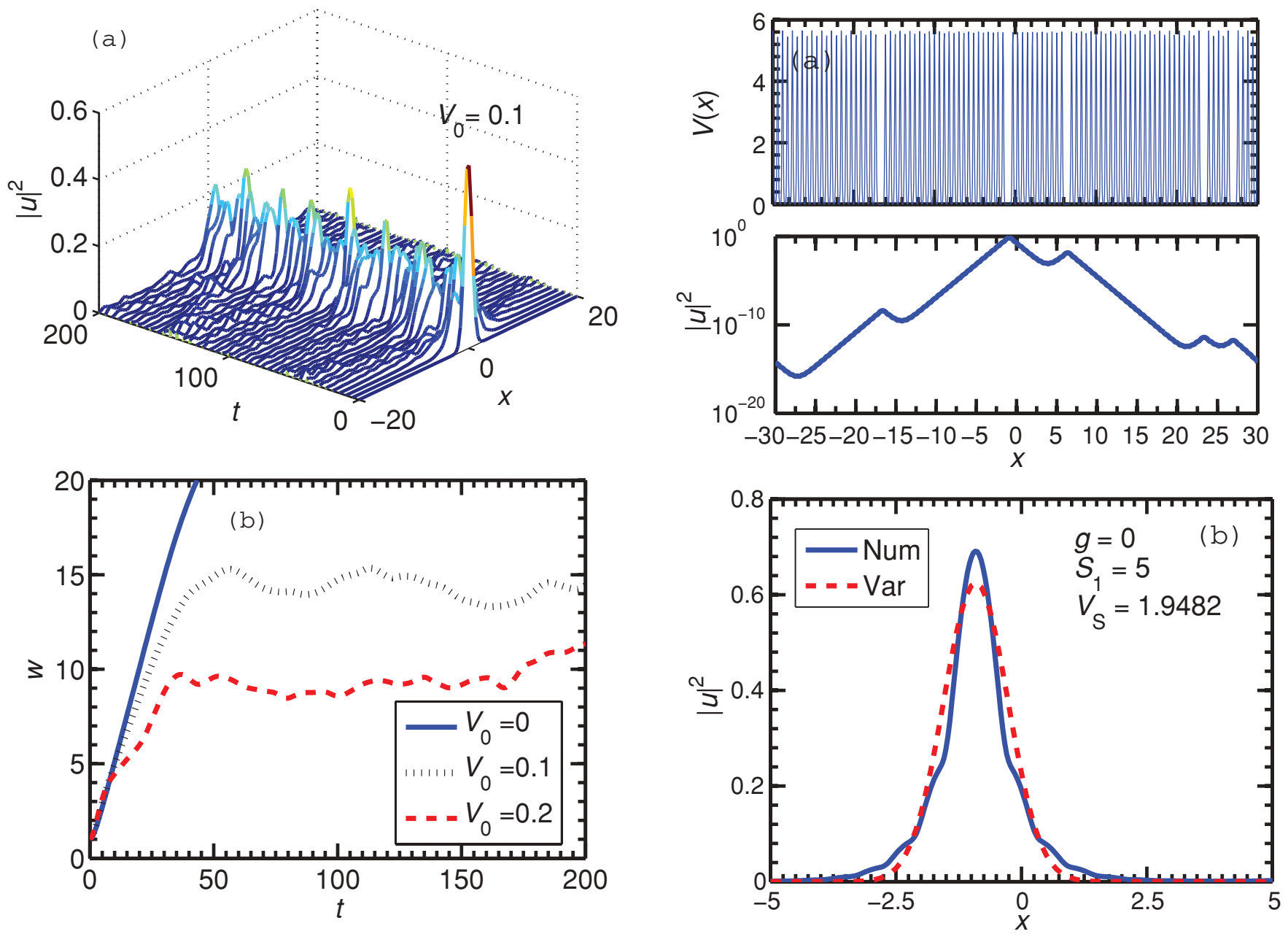

FIG. 5. (Color online) The expansion of a noninteracting BEC in the speckle potential. (a) The time evolution for the density profile $|u|^{2}$ versus $x$ and $t$ of the BEC for $V_{0}=0.1$. (b) The time evolution of the width of the BEC for several $V_{0}$.

initial expansion, the BEC expands quickly. If $V_{0}=0$, the BEC cannot be localized and expands into the whole space (see the solid line). If the potential has weak disorder, however, it will be locked in an appropriate localized state after a certain amount of expansion. After this happens, the system executes breathing oscillation around a mean shape of the localized state and the width remains bounded and does not increase indefinitely with time. The stronger $V_{0}$ is, the shorter the time for the initial expansion is and the smaller is the final width.

\section{B. A disordered cold atom lattice}

Following the model given by Gavish and Castin [10], the disordered cold atom lattice is taken as a set of periodical spikes with a few random vacancies (holes). To numerically generate such a potential, we consider $S=100$ periodic spikes within $x=[-30,30]$ defined by Eq. (3) with $\sigma=0.1$ and $V_{0}=1$. Then we take out a small number $S_{1}$ of spikes whose positions are obtained with the standard MATLAB function RAND. Figure 6(a) shows a typical weakly disordered potential with $S_{1}=5$ (upper panel), and the numerical density profile of the localized BEC in this potential (lower panel) for $g=0$. The modulation of the exponential tail of the density profile is

FIG. 6. (Color online) (a) A typical cold atom lattice (upper panel), and the numerical density profile of the localized state (lower panel) in exponential scale. (b) The numerical (Num) and variational (Var) density profiles $|u|^{2}$ of the localized BEC with disordered potential (a).

clearly visible. In Fig. 6(b) we compare the central part of the numerical density with the variational density for the potential of Fig. 6(a).

In Fig. 7(a) we plot the average spike height $V_{S}$ of Eq. (6) versus $S_{1}$ showing a decrease of $V_{S}$ with $S_{1}$. To understand the effects of the average speckle height on the localized BEC, we numerically calculate the chemical potential of the localized state versus $V_{S}$ of Eq. (6) for four cases. The disordered cold atom lattices corresponding in these cases are created by the same parameters but with different random processes. Although the disordered potentials created by the different random processes possess different local characteristics, the global effects are quite similar. The values and variations of the chemical potential of the system are smaller if $V_{S}$ is smaller, corresponding to strong disorder. In the case of the weak disorder, for example $V_{S}>1.8$, the chemical potentials are very sensitive to the average speckle height. In this case a large $V_{S}$ corresponds to weak disorder in contrast to the speckle potential of Fig. 1, where the same denotes strong disorder.

We also study the dynamics of the localized BEC trapped in a weak disordered cold atom lattice. A harmonically trapped 

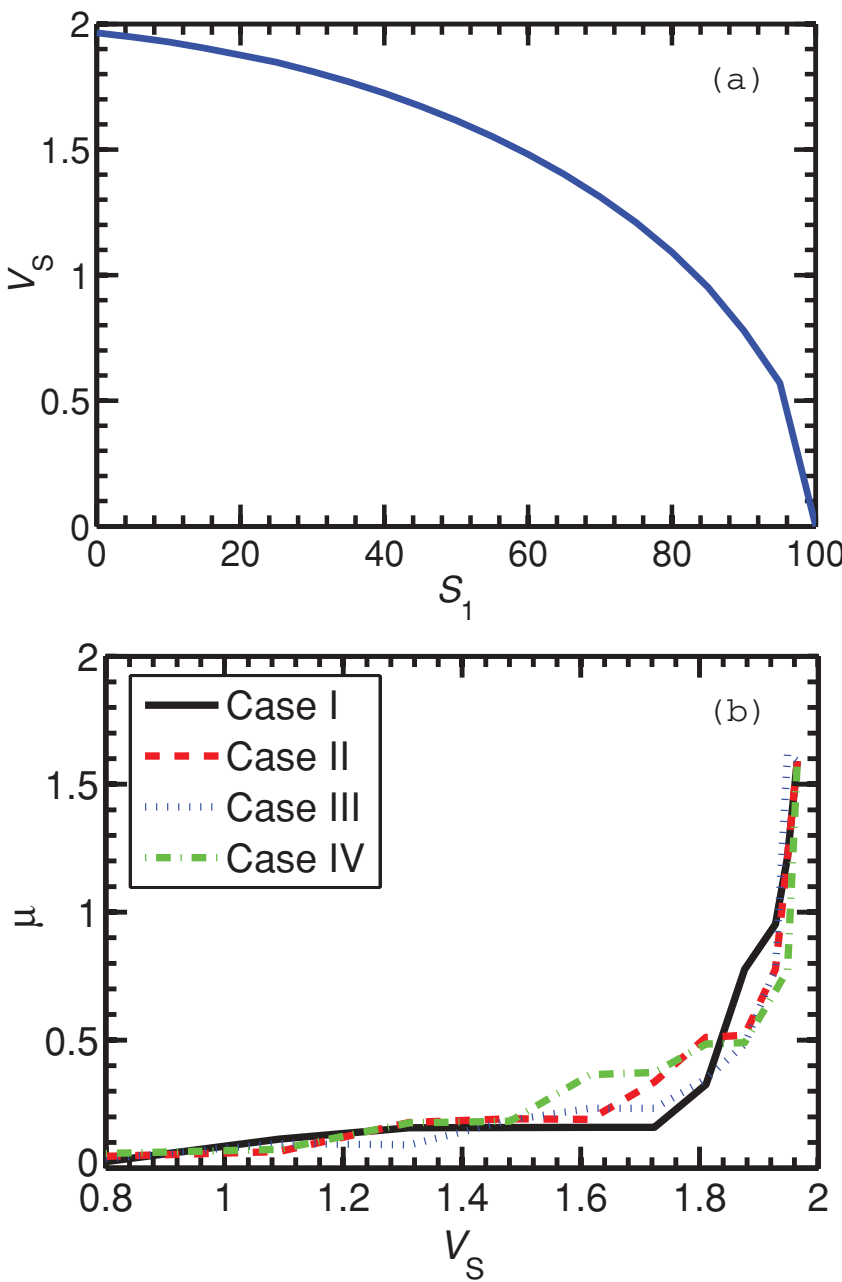

FIG. 7. (Color online) (a) The average spike height of the disordered potential $V_{S}$ of Eq. (6) versus $S_{1}$. (b) The chemical potential of the stationary localized states versus $V_{S}$ in case of four cold atom lattice.

BEC is suddenly released into a weak disordered cold atom lattice shown by Fig. 6(a). The evolution of the density profile and width of the BEC are plotted in Figs. 8(a) and 8(b), respectively. Because of the weak disorder, the BEC can remain as a single fragment during the evolution [see Fig. 8(a)]. If the potential is periodical (corresponding to $S_{1}=0$ ), the BEC cannot be localized. For a small $S_{1}$ the width executes stable breathing oscillation indicating stable localization.

\section{SUMMARY}

In this paper, using the numerical and variational solutions of the time-dependent GP equation, we studied the localization of a noninteracting and weakly interacting BEC in a disordered potential. We considered two models of the disordered potential corresponding to (i) the speckle potential $[1,12]$ and (ii) the disordered cold atom lattice $[10,12]$. These disordered potentials are created by a superposition of narrow spikes randomly distributed in space. In the case of a single BEC fragment, we find that the variational analysis is applicable and produces results in good agreement with numerical simulation. Our investigation shows that the chemical potential (energy)
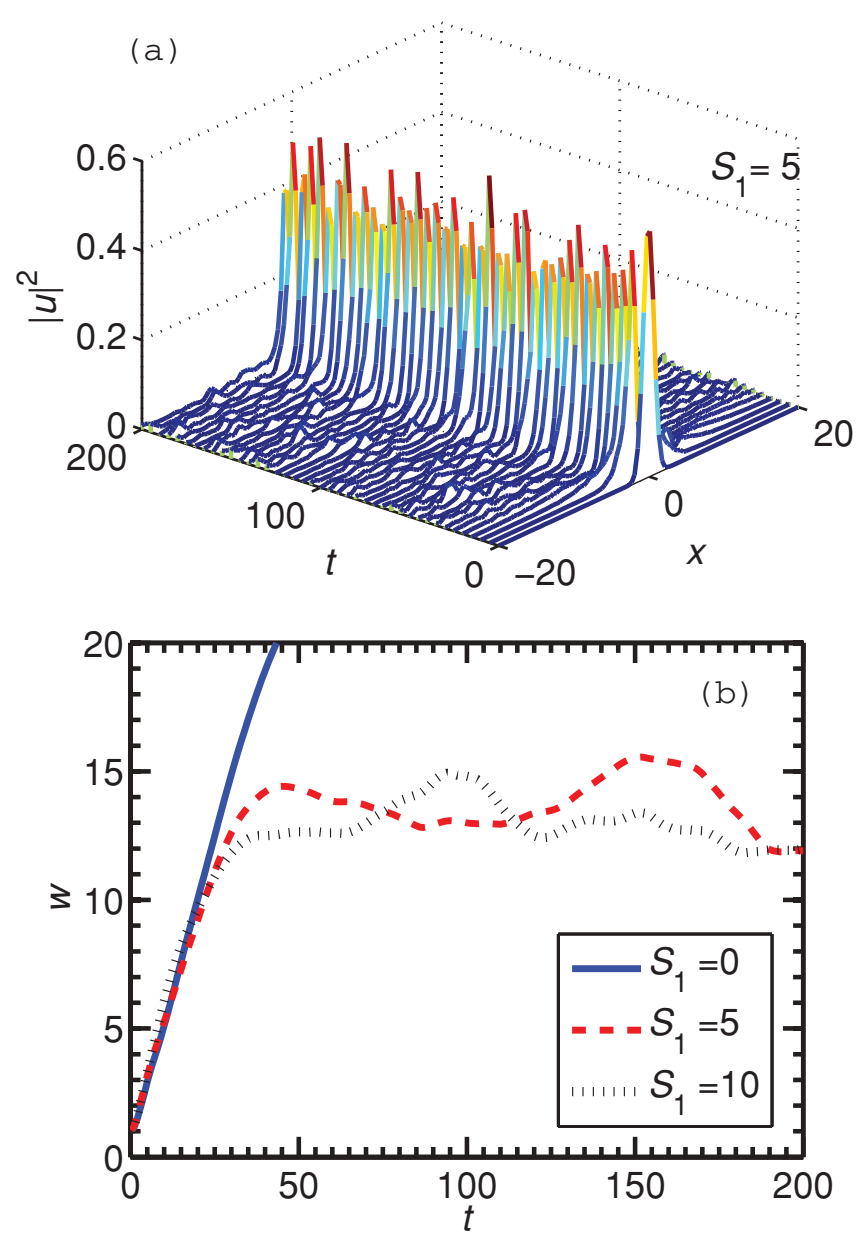

FIG. 8. (Color online) The expansion of a noninteracting BEC in the weak disordered cold atom lattice. (a) The evolution of the density profile of the BEC for $S_{1}=5$. (b) The evolution of the width of the BEC for several $S_{1}$.

of stationary localized BEC is related to the average height of the random potential. The larger the average height is, the larger the chemical potential (energy) is. For a weakly disordered potential, the localized BEC's are found to have an exponential tail as expected in weak Anderson localization [12]. The exponential tails are asymmetric and are modulated by the disordered potential. The effects of nonlinearity on localization are investigated carefully. For a sufficiently weak nonlinearity, the increase of the repulsive nonlinearity leads to an increase of the localization length of the exponential tail and the increase of the chemical potential (energy) of stationary localized BEC. For larger nonlinearity, the localization will be destroyed and the BEC will be in a multifragmented state. We also investigated the expansion of a noninteracting BEC in the disordered potential. We find that the BEC will be locked in an appropriate localized state after an initial quick expansion and will execute breathing oscillation around a mean shape of the localized state when a BEC at equilibrium in the harmonic trap is suddenly released into a potential with weak disorder.

\section{ACKNOWLEDGMENTS}

FAPESP and CNPq (Brazil) provided partial support. 
[1] J. Billy et al., Nature (London) 453, 891 (2008).

[2] G. Roati et al., Nature (London) 453, 895 (2008).

[3] L. Fallani, J. E. Lye, V. Guarrera, C. Fort, and M. Inguscio, Phys. Rev. Lett. 98, 130404 (2007).

[4] A. Eckardt, M. Holthaus, H. Lignier, A. Zenesini, D. Ciampini, O. Morsch, and E. Arimondo, Phys. Rev. A 79, 013611 (2009).

[5] S. Ospelkaus, C. Ospelkaus, O. Wille, M. Succo, P. Ernst, K. Sengstock, and K. Bongs, Phys. Rev. Lett. 96, 180403 (2006).

[6] E. E. Edwards, M. Beeler, T. Hong, and S. L. Rolston, Phys. Rev. Lett. 101, 260402 (2008); J. Chabé, G. Lemarie, B. Gremaud, D. Delande, P. Szriftgiser, and J. C. Garreau, ibid. 101, 255702 (2008); C. Fort, L. Fallani, V. Guarrera, J. E. Lye, M. Modugno, D. S. Wiersma, and M. Inguscio, ibid. 95, 170410 (2005); B. Deissler, M. Zaccanti, G. Roati, C. D'Errico, M. Fattori, M. Modugno, G. Modugno, and M. Inguscio, Nature Phys. 6, 354 (2010).

[7] M. Larcher, F. Dalfovo, and M. Modugno, Phys. Rev. A 80, 053606 (2009).

[8] S. K. Adhikari and L. Salasnich, Phys. Rev. A 80, 023606 (2009); Y. S. Cheng and S. K. Adhikari, ibid. 81, 023620 (2010); S. K. Adhikari, ibid. 81, 043636 (2010).

[9] M. Modugno, New J. Phys. 11, 033023 (2009); D. J. Boers, B. Goedeke, D. Hinrichs, and M. Holthaus, Phys. Rev. A 75, 063404 (2007); G. Roux, T. Barthel, I. P. McCulloch, C. Kollath, U. Schollwock, and T. Giamarchi, ibid. 78, 023628 (2008); T. Roscilde, ibid. 77, 063605 (2008); X. Cai, S. Chen, and Y. Wang, ibid. 81, 023626 (2010); X. Deng, R. Citro, E. Orignac, and A. Minguzzi, Eur. Phys. J. B 68, 435 (2009); M. Albert and P. Leboeuf, Phys. Rev. A 81, 013614 (2010); S. Aubry and G. André, Ann. Isr. Phys. Soc. 3, 133 (1980).

[10] U. Gavish and Y. Castin, Phys. Rev. Lett. 95, 020401 (2005).

[11] P. Massignan and Y. Castin, Phys. Rev. A 74, 013616 (2006).

[12] L. Sanchez-Palencia, D. Clément, P. Lugan, P. Bouyer, and A. Aspect, New J. Phys. 10, 045019 (2008); L. Sanchez-Palencia and M. Lewenstein, Nature Phys. 6, 87 (2010).

[13] N. Cherroret and S. E. Skipetrov, Phys. Rev. A 79, 063604 (2009); T. Nattermann and V. L. Pokrovsky, Phys. Rev. Lett. 100, 060402 (2008).

[14] G. Kopidakis, S. Komineas, S. Flach, and S. Aubry, Phys. Rev. Lett. 100, 084103 (2008); A. S. Pikovsky and D. L.
Shepelyansky, ibid. 100, 094101 (2008); I. García-Mata and D. L. Shepelyansky, Phys. Rev. E 79, 026205 (2009); S. Flach, D. O. Krimer, and Ch. Skokos, Phys. Rev. Lett. 102, 024101 (2009); Ch. Skokos, D. O. Krimer, S. Komineas, and S. Flach, Phys. Rev. E 79, 056211 (2009); T. Paul, M. Albert, P. Schlagheck, P. Leboeuf, and N. Pavloff, Phys. Rev. A 80, 033615 (2009).

[15] B. Damski, J. Zakrzewski, L. Santos, P. Zoller, and M. Lewenstein, Phys. Rev. Lett. 91, 080403 (2003); T. Schulte, S. Drenkelforth, J. Kruse, W. Ertmer, J. Arlt, K. Sacha, J. Zakrzewski, and M. Lewenstein, ibid. 95, 170411 (2005); L. Sanchez-Palencia and L. Santos, Phys. Rev. A 72, 053607 (2005).

[16] L. Sanchez-Palencia, D. Clement, P. Lugan, P. Bouyer, G. V. Shlyapnikov, and A. Aspect, Phys. Rev. Lett. 98, 210401 (2007); D. Clément et al., New J. Phys. 8, 165 (2006).

[17] J. E. Lye, L. Fallani, M. Modugno, D. S. Wiersma, C. Fort, and M. Inguscio, Phys. Rev. Lett. 95, 070401 (2005).

[18] D. Clément, A. F. Varon, M. Hugbart, J. A. Retter, P. Bouyer, L. Sanchez-Palencia, D. M. Gangardt, G. V. Shlyapnikov, and A. Aspect, Phys. Rev. Lett. 95, 170409 (2005).

[19] L. Fallani, C. Fort, and M. Inguscio, Adv. At. Mol. Opt. Phys. 56, 119 (2008).

[20] H. Gimperlein, S. Wessel, J. Schmiedmayer, and L. Santos, Phys. Rev. Lett. 95, 170401 (2005).

[21] Ph. W. Courteille et al., J. Phys. B 39, 1055 (2006).

[22] S. Palpacelli and S. Succi, Phys. Rev. E 77, 066708 (2008); M. Modugno, Phys. Rev. A 73, 013606 (2006); E. Akkermans, S. Ghosh, and Z. H. Musslimani, J. Phys. B 41, 045302 (2008)

[23] C. A. G. Buitrago and S. K. Adhikari, J. Phys. B 42, 215306 (2009); L. Salasnich, A. Parola, and L. Reatto, Phys. Rev. A 65, 043614 (2002).

[24] P. J. Y. Louis, E. A. Ostrovskaya, C. M. Savage, and Y. S. Kivshar, Phys. Rev. A 67, 013602 (2003).

[25] V. M. Pérez-García, H. Michinel, J. I. Cirac, M. Lewenstein, and P. Zoller, Phys. Rev. A 56, 1424 (1997); S. K. Adhikari and B. A. Malomed, ibid. 79, 015602 (2009); Y. S. Cheng, R. Z. Gong, and H. Li, Opt. Express 14, 3594 (2006); B. A. Malomed, Prog. Opt. 43, 69 (2002). 